\title{
Using Plasma Focused Ion Beam Microscopy to Characterize 3D Structure and Porosity of OPC Mortar
}

\author{
Peng Dong ${ }^{1}$, Ali Allahverdi ${ }^{2}$, Hui Yuan ${ }^{1}$ and Nabil D. Bassim ${ }^{1 *}$ \\ 1. Department of Materials Science and Engineering and Canadian Centre for Electron Microscopy, \\ McMaster University, Hamilton, ON, Canada. \\ 2. School of Chemical Engineering, Iran University of Science and Technology, Tehran, Iran. \\ * Corresponding author: bassimn@mcmaster.ca
}

Ordinary Portland cement (OPC) and its concretes have been used in construction industry for more than 100 years. The permeability and corresponding transport properties of OPC mortar or concrete are largely determined by pore characteristics, including porosity, connectivity, and pore throat size. Many techniques such as scanning electron microscopy (SEM), or atom force microscopy (AFM) can provide information even at nanometer range. But they are limited to a $2 \mathrm{D}$ projection, which can be misleading for quantitification. Transmission electron microscopy (TEM) tomography and atom probe tomography allow 3D analysis of structure at even higher resolutions. The sizes of volume of interest (VOI) in above techniques are not representative though, and any meaningful measurement would require a large amount of repetitive experiments. The X-ray computed tomography (CT) is applicable to bulk samples, while the resolution is limited to the micrometer scale and relies on absorption contrast which gives little information about chemistry. This is not enough to analyze nanoscale pores distribution and materials chemistry. Finally, mercury intrusion porosimetry (MIP), a traditional technique used in cementitious materials, enables the analysis of pore size distribution. However, the results possess an overestimation of small pores due to the ink-bottle effect [1].

Focused ion beam nanotomography (FIB-nt) allows insights into 3D structure with relatively large volume and nanoscale resolution, which is suitable to characterize pore systems at multiple length scales. By alternating imaging a block face of material, following by slicing that material using an ion beam, a serial sectioning tomography method is possible. New plasma sources that use $\mathrm{Xe}^{+}$ions produce a higher milling rate than traditional Ga liquid metal ion sources and make 3D tomography with volumes up to hundreds of microns in higher milling rate with better surface finish. $[2,3]$. Using a $\mathrm{Xe}^{+}$Plasma FIB at the Canadian Center of Electron Microscopy (CCEM) at McMaster University, we conducted 3D tomography on an OPC mortar specimen using Plasma FIB and showed the feasibility of acquiring large volume, high resolution tomograms of cementitious materials. Figure 1 (a) showed a backscattered electron (BSE) image, in which the slice contained anhydrous cement, paste, sand particle, cracks and pores. Curtaining removal (Fig. 1 (b)) was conducted using a Fourier-based linear optimization model computing [4]. By applying thresholding, the crack and pore networks were separated in Fig. 2. More complex segmentation allows the visualization and quantification of cementitious materials and the start of mesoscale characterization of complex geological and construction materials using plasma FIB. 
References:

[1] L Holzer in "Review of FIB-tomography", ed. I Utke, (Oxford University Press, New York) p. 410.

[2] N Bassim, K Scott, and LA Giannuzzi, MRS Bulletin 39 (2014), p. 317.

[3] TL Burnett et al., Ultramicroscopy 161 (2016), p. 119.

[4] CC Schankula, Anand, and N Bassim, Computing and Software Report (2018).
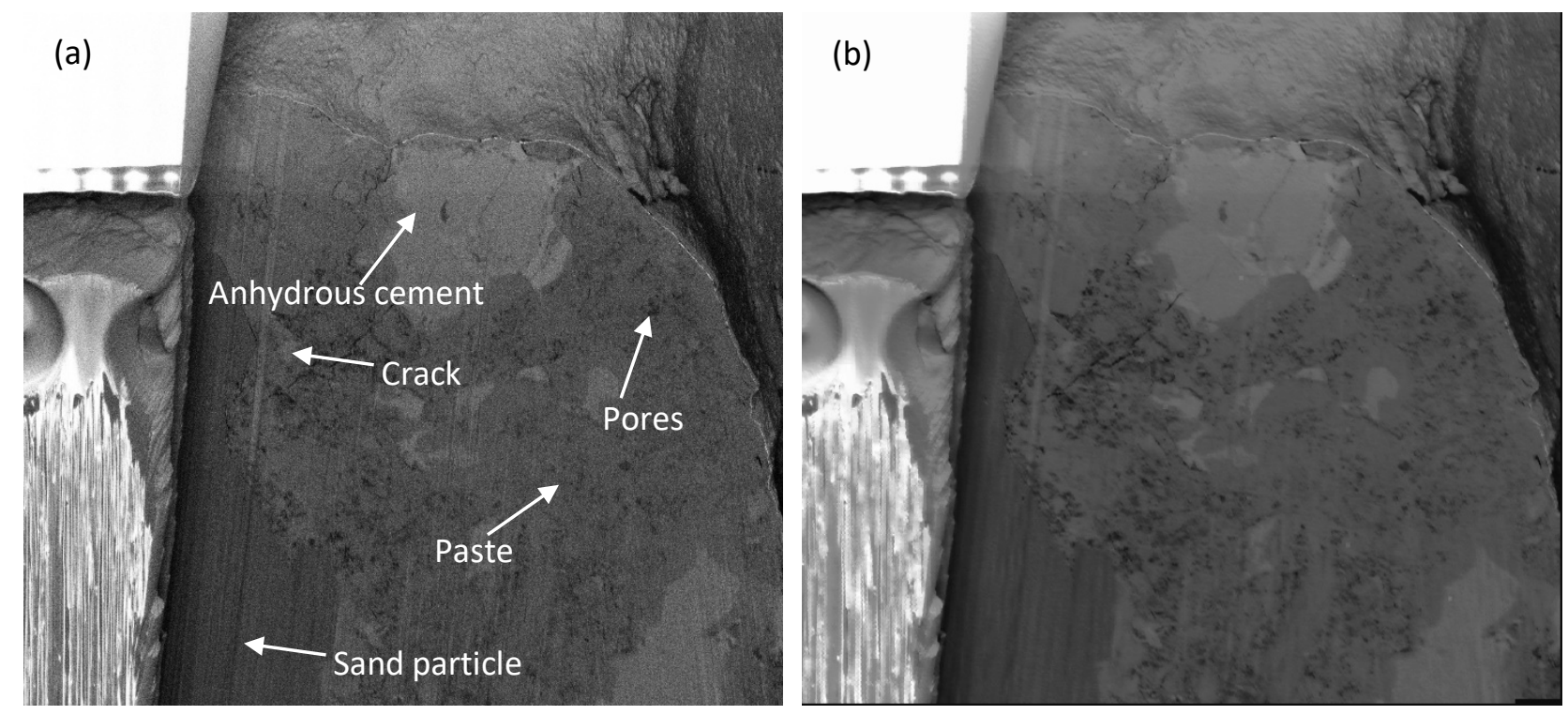

Figure 1. a) TLD image of one slice $(153.6 \mu \mathrm{m} \times 140 \mu \mathrm{m})$ showing BSE contrast by PFIB, and b) The same slice after median filtering and curtaining removal
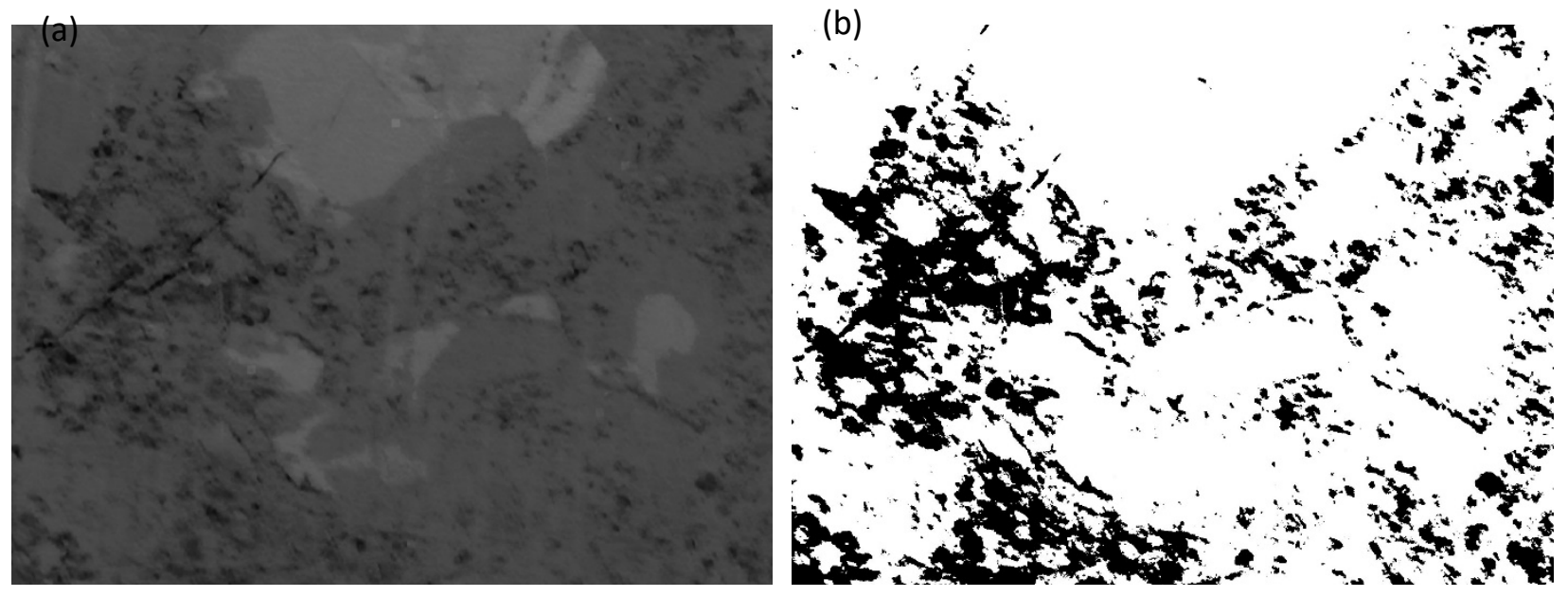

Figure 2. Segmentation of single slice after cropping $(40.3 \mu \mathrm{m} \times 29.6 \mu \mathrm{m})$ applying simple thresholding methods 